\title{
DEVELANDO LOS DETERMINANTES DE LA DESIGUALDAD DEL INGRESO EN CHILE: ESTUDIO EMPIRICO REGIONAL*
}

\author{
REVEALING THE DETERMINANTS OF INCOME INEQUALITY \\ IN CHILE: A REGIONAL EMPIRICAL STUDY
}

\section{MICHELLE MIERES BREVIS** \\ Universidad de Alicante}

\begin{abstract}
The study aims to answer which are the determinants of income inequality in Chile, from a regional perspective. The empirical analysis (1990-2016) shows that in Chile the relationship between the GDP per capita and the Gini does not have the traditional form of the inverted Kuznets $U$, but rather, the behavior is the inverse, same observed in countries such as United States and the United Kingdom. It is revealed that, initial values of income; the economic activity of the region; the concentration of indigenous population and human capital are important and robust determinants of income inequality in Chile.
\end{abstract}

Keywords: Inequality, income inequality, economic growth, economic development.

JEL Classification: B23, C3, D31, D63, F43.

* Este artículo es parte de mi trabajo de investigación en el programa de Doctorado Interuniversitario DEcIDE, en la Universidad de Alicante (Carretera de San Vicente del Raspeig, s/n, 03690 San Vicente del Raspeig, Alicante, España. Teléfono: 96590 3609). El trabajo se ha beneficiado de los comentarios y sugerencias de mi directora de tesis María Dolores Guilló y de los asistentes al Seminario Novel 2018, realizado en la Universidad Miguel Hernández de Elche, en el cual presenté este estudio. El trabajo también fue expuesto en el Seminario "Miradas de la Desigualdad en Chile y La Araucanía", en la Universidad de La Frontera, Temuco-Chile, en enero de 2019. Correo: mmb124@alu.ua.es

** Este trabajo es posible gracias al apoyo de CONICYT PFCHA/DOCTORADO BECAS CHILE/2017-72180047. 


\section{Resumen}

El estudio busca responder cuáles son los determinantes de la desigualdad del ingreso en Chile, desde la perspectiva de sus regiones. El análisis empírico (1990-2016) demuestra que en Chile la relación entre el PIB per cápita y el Gini no tiene la tradicional forma de la U invertida de Kuznets, sino más bien, el comportamiento es el inverso, mismo observado en países como EE.UU. y Reino Unido. Se revela que, valores iniciales del ingreso; la actividad económica regional; la concentración de población indígena y el capital humano, son importantes y robustos determinantes de la desigualdad del ingreso en Chile.

Palabras clave: Desigualdad, desigualdad del ingreso, crecimiento económico, desarrollo económico.

Clasificación JEL: B23, C3, D31, D63, F43.

\section{INTRODUCCION}

Chile es un país en vías de desarrollo con importantes resultados en materia de crecimiento económico, sobre todo en las últimas dos décadas. Es el primer país Latinoamericano invitado a ser parte de la Organización para la Cooperación y el Desarrollo Económico (OCDE). No obstante, los buenos indicadores macroeconómicos no han podido mitigar el persitente y elevado efecto de la desigualdad del ingreso. Actualmente, Chile es el segundo país más desigual entre los miembros de la OCDE (medida a través del índice de Gini antes y después de impuestos y transferencias). La desigualdad del país es intra e interregional, los ingresos del 20\% de la población más rica son 10 veces mayores que los del quintil más pobre (OCDE, 2018), siendo sorprendentemente mayor que el promedio de los países de la OCDE. "En Chile estamos viendo un fenómeno de reducción de las brechas que ya tiene algunos años... la dirección emprendida es la correcta, pero el nivel sigue siendo inaceptable desde el punto de vista de nuestras ambiciones respecto de una sociedad más igualitaria" (Extracto de El Mercurio del día 24 de octubre de 2013 sobre el Discurso de Angel Gurría, en la $32^{a}$ reunión anual de la Red de Altos Funcionarios de Centros de Gobierno).

Desde hace décadas, existe consenso en la literatura económica, en que un país no debe ser evaluado únicamente por sus indicadores económicos de productividad, sino también por su capacidad para reducir la pobreza y la desigualdad, conceptos entendidos como males para una sociedad (Gasparini, Cicowiez y Sosa, 2014). Las actuales inequidades económicas y sociales del país generan constante rechazo y levantamientos por parte de la sociedad, por ello, un objetivo clave para cada 
Gobierno debe ser limitar al máximo las brechas que separan a los chilenos, de forma de tener una sociedad más justa y potenciar el crecimiento y desarrollo en cada uno de los territorios. Es imperante que el crecimiento en Chile sea inclusivo y se sostenga en el tiempo. Además, el hecho de obtener un ingreso insuficiente es consecuencia de las desigualdades estructurales de un sistema económico, por tanto "la eliminación de la pobreza requiere reformas estructurales e institucionales, económicas y sociales, que erradiquen las desigualdades que reproducen la pobreza" (Macías, 2014, pp. 121).

Este artículo busca responder cuáles son los determinantes de la desigualdad del ingreso, desde la perspectiva de sus regiones. Es en lo último donde radica la novedad del estudio, ya que contribuye al tema de la desigualdad, anteriormente estudiado en Chile, pero desde un punto de vista regional. Para lograr el objetivo se trabaja con la base de datos construida en el trabajo anterior de Mieres (2019). La segunda sección muestra los principales factores mencionados en la literatura por su impacto en la desigualdad del ingreso. Los resultados del análisis empírico aplicado a las regiones de Chile están en la sección tres. Finalmente se presentan las principales conclusiones y limitaciones del estudio.

El trabajo es un esfuerzo necesario para la sociedad chilena, de reflexionar sobre los factores que promueven mayormente las desigualdades, y es un llamado de atención para las autoridades a conocer los efectos del actual modelo de crecimiento económico de Chile sobre la desigualdad. Invita a enfocar la inversión pública en todos aquellos factores que permiten reducir las brechas entre ricos y pobres, logrando de esta manera el anhelado desarrollo inclusivo. En Chile, las brechas sociales y económicas crean malestar por parte de la ciudadanía y descontento hacia las autoridades públicas, generando épocas de constantes manifestaciones, donde la población exige un crecimiento y desarrollo para todos.

La metodología para el desarrollo de esta investigación consiste en un análisis econométrico, el cual sigue la metodología utilizada en los estudios de Forbes (2000) y Barro (2000) y conjuga las variables que, de acuerdo a Kuznets (1955), Barro (2000) y los autores que se mencionarán a lo largo de la Sección 2, determinan la desigualdad del ingreso, siendo la variable dependiente el índice de Gini. La información recopilada para el desarrollo de este trabajo, se ha obtenido de la base de datos construida en Mieres (2019) ${ }^{1}$.

El análisis de datos de panel, del período 1990-2016, revela una relación cuadrática entre el PIB per cápita y el Gini, pero no como lo plantea Kuznets, sino más bien a la inversa. Los altos ingresos disminuyen las desigualdades, pero la tendencia negativa

1 Este trabajo utilizó fuentes secundarias desde distintas bases de datos mundiales y nacionales. A nivel país, se utilizó principalmente, la Encuesta de Caracterización Socioeconómica Nacional (CASEN) del Ministerio de Desarrollo Social y datos de productividad del Banco Central de Chile. Asimismo, se contactó con diferentes Instituciones, como el Instituto Nacional de Estadísticas, a quien se solicitaron datos antiguos poblacionales y vitales no disponibles en la web. 
se convierte en positiva a partir de un cierto nivel de renta. Esta relación se observa también en países como Estados Unidos, Reino Unido, Costa Rica e India (Deininger y Squire, 1998).

De las distintas estimaciones, se infiere que dentro de los factores que influyen mayormente en las desigualdades regionales, destacan: los ingresos iniciales (PIB per cápita); la actividad económica principal de la región; los indicadores de salud; la educación (particularmente la oferta de educación superior) y la concentración de la población indígena. Mejorar el capital humano, aumentando las oportunidades de acceso a la educación y a un sistema de salud de calidad, así como redoblar los esfuerzos para disminuir la brecha entre la población indígena y no indígena, son medidas necesarias para alcanzar un mayor nivel de desarrollo y en consecuencia mejorar la calidad de vida de la población chilena.

Asimismo, se enfatiza en la importancia de aumentar las rentas de la población de menores ingresos, ya que se demuestra que períodos de tiempo en que el ingreso de los ricos aumenta proporcionalmente más que el de los pobres, son épocas donde aumenta la desigualdad. Por su lado, en períodos donde los ingresos de las familias más pobres son los que proporcionalmente tienen un mayor incremento, las desigualdades se reducen. Desmintiendo, acorde con distintos autores (Arndt, 1983; Guillén, 2004 y Piketty, 2014) la teoría del “goteo", la cual postula que, favorecer a los estratos más ricos de la población, tiende a favorecer al resto de la sociedad, a través de un goteo que llega a las personas menos favorecidas, en consecuencia la desigualdad se reduce.

Finalmente, se prueba la robustez de los resultados utilizando como variable dependiente una medida de desigualdad distinta al Gini, el ratio 20/20. Los resultados utilizando ambas variables son similares, sin embargo, utilizando el ratio 20/20, los coeficientes estimados son mayores y los ajustes de los modelos se incrementan.

"En los últimos diez años la investigación ha empezado a poner de manifiesto lo negativa que resulta la desigualdad para la sociedad. Resulta mala incluso para los de arriba, que se convierten en personas diferentes -más endiosadas- gracias a ella" (Joseph Stiglitz, Premio Novel de Economía 2001).

\section{DETERMINANTES DE LA DESIGUALDAD DEL INGRESO}

En el trabajo anterior de Mieres (2019), se develó la gran heterogeneidad presente entre las regiones chilenas, y la gran desigualdad económica y social ${ }^{2}$. Es lamentable ver que a nivel de desigualdad del ingreso, las regiones del sur suelen encontrarse

2 La Tabla del Anexo D resume los resultados de los indicadores presentados en el trabajo de Mieres (2019). 
en una posición desfavorecida versus las regiones nortinas. Los territorios más desiguales, son también aquellos con menor PIB per cápita y menores indicadores de desarrollo. Además se concluyó que existe convergencia absoluta en términos de PIB per cápita y desigualdad del ingreso. En promedio, las regiones más pobres tienden a crecer más que aquellas más ricas. Asimismo, las regiones más desiguales inicialmente, son las que tienden a reducir más rápido este indicador. A pesar de lo anterior, la desigualdad de la renta es alta y persistente en el tiempo. Estos resultados motivan el análisis econométrico que busca exponer los principales determinantes de la desigualdad del ingreso en Chile.

Es amplia la literatura que relaciona el crecimiento económico con la desigualdad de un territorio. Comenzando por Kuznets (1955), cuya teoría sostiene que cuando una economía comienza a crecer, también lo hacen las desigualdades, hasta llegar a un cierto punto de desarrollo, donde luego a pesar del mayor crecimiento económico, las desigualdades van disminuyendo, tomando la forma de una $\mathrm{U}$ invertida ${ }^{3}$.

Deininger y Squire (1998), en una investigación que buscaba confirmar o refutar la hipótesis de Kuznets, realizaron un análisis con datos longitudinales de 49 países del mundo. En la mayoría de los casos no encontraron una relación significativa entre ingreso per cápita y desigualdad. En cinco de los países encontraron una relación que confirma la hipótesis de la U invertida (Brasil, México, Hungría, Trinidad y Filipinas), mientras que en cuatro de ellos (Estados Unidos, Reino Unido, Costa Rica e India), encontraron evidencia que la contradice. Los autores interpretan sus resultados como una indicación de que no hay una ley universal inamovible, sino que más bien, los cambios en los ingresos y la desigualdad, son afectados por las condiciones iniciales de los países y posiblemente por las políticas (Deininger y Squire, 1998, pp. 261).

En su trabajo Barro (2000) encuentra una regularidad empírica clara de la teoría de Kuznets. Sin embargo, concluye que esta relación (el PIB per cápita) no explica la mayor parte de las variaciones en la desigualdad entre países o a lo largo del tiempo. Por ello, en su estudio incluye variables como el nivel educacional, la apertura económica y el índice de democracia, como determinantes de la desigualdad de la renta.

3 La explicación de su teoría radica en que los países más pobres dependían principalmente de la agricultura, actividad con baja productividad, pero baja desigualdad. A partir de los procesos de migración de trabajadores del campo a la ciudad, el sector más productivo, el industrial, empezó a recibir trabajadores no calificados y con bajos salarios, promoviendo una elevada inequidad, pero acompañada de un incremento de la producción (parte positiva de la curva). En la siguiente fase, hay una gran cantidad de trabajadores ubicados en el sector industrial y una reducción del número de trabajadores en el sector agrícola, permitiendo un aumento de sus salarios relativos (por la escasa oferta). Aquí, la relación entre crecimiento y desigualdad se vuelve negativa. 
Solimano y Torche (2008) realizaron un estudio para Chile entre los años 1987 y 2003, encontrando una relación no lineal entre el PIB per cápita y el Gini (medido para los ingresos de las personas y los hogares). Este estudio valida la hipótesis de la U invertida de Kuznets, sin embargo, no observan causalidad, ya que estudian el mismo período de tiempo tanto en la variable dependiente, como en las independientes. Otras variables significativas, correlacionadas con la desigualdad, incluidas en este estudio son: la escolaridad promedio (relación negativa), nivel de ocupación por región (relación positiva), ingreso autónomo de los hogares (relación positiva), entre otras.

Por su parte, la Nueva Geografía Económica plantea que las desigualdades entre regiones pueden mantenerse en el tiempo e incluso crecer junto con la economía de un país, ya que existe un centro que aprovecha los mayores beneficios del crecimiento, mientras que las regiones de la periferia pierden esa oportunidad (Merchand, 2017).

La desigualdad del ingreso está estrechamente relacionada con la desigualdad social. En Chile, mayores ingresos son consecuentes con mejores oportunidades de acceso a un sistema de educación y de salud de calidad ${ }^{4}$. Las personas que gozan de mayores ingresos tienen la posibilidad de ir a mejores escuelas, asistir a clases particulares y extracurriculares, ir a mejores centros médicos e incluso tomar mejores medicinas, que una persona pobre. Estas mismas condiciones sociales y otras medioambientales y de seguridad, son determinantes para el futuro nivel de ingresos de las personas, por tanto, peores condiciones sociales pueden aumentar las desigualdades del ingreso.

Contreras (1999) plantea que la educación es la variable más importante para explicar la desigualdad del ingreso salarial, en segundo lugar están los años de experiencia, ambas, en conjunto, forman el capital humano. En su trabajo el autor concluye que, "la educación determina la distribución del ingreso a través del cambio en su retorno, el cual a su vez es explicado por cambios en la demanda por trabajo calificado" (pp. 325), esto quiere decir que para reducir la desigualdad es necesario invertir más en educación, lo que aumentará la oferta de trabajo calificado, traduciéndose en aumento de ingresos para estas personas y a la vez reducirá los retornos de este grupo y la desigualdad. Asimismo, la educación es considerada una de las principales herramientas para el desarrollo, al mejorar la productividad y la innovación, reduciendo así, las brechas socioeconómicas de una sociedad.

El estudio de panel de Barro (2000), que analiza los determinantes de la desigualdad, incluye como variable explicativa el promedio de años de estudios de personas de 15

4 En Chile, el sistema de educación pública (principalmente educación básica y media) debe mejorar en calidad para ser competitivo con el sector privado. Por su parte, el sistema de salud presenta inconvenientes, principalmente por la falta de atención oportuna. 
años o más en tres niveles: primario, secundario y superior. Concluye que la educación primaria está relacionada de manera negativa y significativa con la desigualdad, la educación secundaria se relaciona negativamente (aunque no significativamente) con la desigualdad, y que la educación superior está relacionada positiva y significativamente con la desigualdad.

Por otro lado, la pobreza y la mala salud son fenómenos interrelacionados. De acuerdo con Wagstaff (2002), a nivel sanitario, los países pobres tienden a presentar peores resultados que los países ricos, y a su vez, dentro de cada país ocurre la misma situación. El autor plantea que la pobreza provoca una salud deficiente por distintas carencias sufridas por las personas más pobres y, a su vez, existe pérdida de ingresos cuando se cuenta con mala salud. Por tanto, la mala salud se traduce en que los pobres sigan siendo pobres. En consecuencia la mala salud aumenta las desigualdades del ingreso. En el ámbito sanitario Chile ha presentado gran progreso en las últimas décadas, con cifras comparables con países desarrollados en cuanto a mortalidad infantil y general. Sin embargo, al igual que con el crecimiento, estos logros no son inclusivos, es decir, no benefician a toda la población de la misma manera, existiendo diferencias a nivel regional.

En distintos estudios, la tasa global de fertilidad cobra relevancia al evaluar su efecto sobre la desigualdad de los ingresos. En 1996, Perotti concluye que las sociedades más igualitarias tienen bajas tasas de fertilidad. Larrañaga y Herrera (2008), quienes estudiaron los recientes cambios en la desigualdad y la pobreza en Chile, concluyen que las tasas de fertilidad tienden a converger entre las mujeres de distintos niveles socioeconómicos, "mientras que a inicios de los sesenta la población de menor nivel socioeconómico tenía alrededor de 2,5 hijos más que la población de mayor nivel socioeconómico, a inicios de 2000 la brecha había disminuido a 1,0 hijo". Chile presenta un bajo índice de fertilidad, siendo de 1,8 nacimientos por mujer, cayendo por debajo de la tasa de reemplazo (Mieres, 2019). Las familias con más hijos, que habitualmente son las más pobres, deben distribuir sus ingresos entre un mayor número de personas, incrementando los niveles de desigualdad. Por tanto, la convergencia señalada por Larrañaga y Herrera (2008) debiera contribuir a una reducción de la brecha entre ricos y pobres.

\section{FACTORES QUE IMPACTAN EN LA DESIGUALDAD DEL INGRESO: ANALISIS EMPIRICO}

El retrato de las desigualdades chilenas obtenido en Mieres (2019) ha presentado distintas variables que, de acuerdo a la literatura expuesta, tienen un efecto sobre la desigualdad, siendo una de ellas el PIB per cápita. Datos como el PIB per cápita, índice de Gini 2013-2015, índice 10/10 regional e índice 20/20 regional fueron calculados por la autora (el primero con base en datos del Banco Central de Chile 
e INE, y los demás en base a la Encuesta CASEN). Para obtener una base de datos del PIB per cápita más amplia, se utilizó el método de la tasa de variación (Correa et al., 2002), de forma tal de unir una serie de datos del PIB por región, a precios constantes, base 1986, 1996, 2003 y el PIB volumen a precios del año anterior encadenado (series enlazadas) referencia 2013, llevando los valores a este último año. Igualmente, se recurrió al contacto con instituciones del Gobierno, para solicitar datos no disponibles libremente en la web, principalmente al Instituto Nacional de Estadísticas de Chile 5 .

Esta sección busca responder empíricamente cuáles son los determinantes de la desigualdad del ingreso en Chile. Tomando en cuenta que el número de regiones de un país por lo general es pequeño, es común que los modelos regionales se planteen mayoritariamente de forma multiecuacional (Martín-Guzmán, 1988). Es por ello que se opta por un análisis con datos de panel para responder a la interrogante planteada. Debido a la disponibilidad de datos, se considera un panel con índices de Gini observados en el horizonte temporal ${ }^{6}$ 1990-2015 (dividido en cinco períodos) ${ }^{7}$ y las distintas variables descritas en la sección anterior. Utilizando este criterio se trabaja con 15 regiones de Chile, obteniendo 69 observaciones.

El Gráfico 1 muestra la relación entre el LN (PIB per cápita) y el índice de Gini. Se observa que el comportamiento no se adecua a la teoría de la U invertida de Kuznets, más bien el comportamiento parece ser el contrario. A mayor nivel de ingreso las desigualdades regionales disminuyen al principio, lo cual es un proceso deseable para un crecimiento económico inclusivo. Sin embargo, la tendencia negativa se convierte en positiva a partir de un cierto nivel de renta, alertando que la actual modalidad de crecimiento en Chile, exigiría sacrificar la equidad en aras de obtener una mayor eficiencia. Este mismo comportamiento ha sido encontrado para Estados Unidos, Reino Unido, Costa Rica e India, en el trabajo de Deininger y Squire (1998).

5 Otras Instituciones a las cuales se pidió información a través del Portal de Transparencia de Chile fueron: Servicio Nacional de Aduanas; Ministerio del Medio Ambiente; Ministerio de Educación; Subsecretaría de Salud Pública; Subsecretaría de Desarrollo Regional; Subsecretaría de Servicios Sociales; Agencia de Promoción de la Inversión Extranjera y el Consejo Nacional de Educación.

6 El índice de Gini se obtiene hasta 2015, pero de otras variables, como PIB per cápita, se obtienen observaciones hasta 2016.

7 Los períodos son $\mathrm{t}=1: 1990-1994 ; \mathrm{t}=2: 1996-2000 ; \mathrm{t}=3: 2003-2006 ; \mathrm{t}=4: 2009-2013$ y $\mathrm{t}=5: 2013$ 2016. A cada uno de estos períodos se le asigna un valor observado de Gini final y un valor del resto de variables al principio del período. Por ejemplo, para el segundo período (1996-2000), el Gini final corresponde al año 2000, mientras que el PIB per cápita inicial y demás variables de control corresponden al período anterior. En la práctica, se hace referencia, en este caso, al año 1996. La división, en estos períodos de tiempo, se debe a la disponibilidad de datos, haciendo referencia a los años en que se realizó la Encuesta CASEN. 


\section{GRAFICO 1}

\section{DISPERSION LN (PIB PER CAPITA) E INDICE DE GINI} 1990-2016

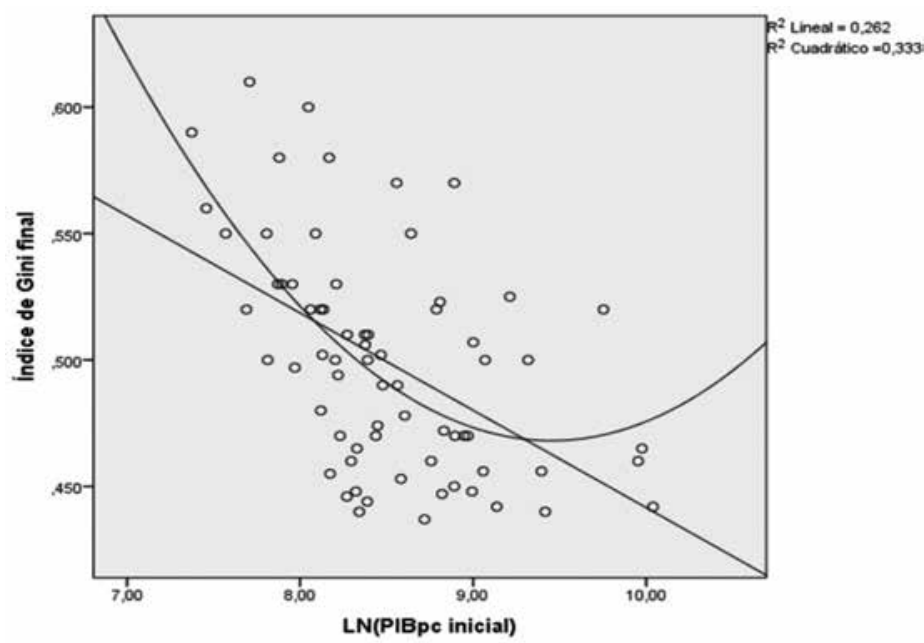

Fuente: Elaboración propia.

El modelo a estimar, sigue la metodología de Forbes $(2000)^{8}$ y Barro (2000), y conjuga las variables que de acuerdo a Kuznets (1955), Barro (2000) y los autores mencionados a lo largo de la Sección 2, determinan la desigualdad, siendo la variable dependiente el índice de Gini. El modelo general queda expresado de la siguiente manera:

$$
\operatorname{GINI}_{i, t}=\alpha+\beta_{1} \operatorname{INGRESO}_{i, t-1}+B X_{i, t-1}+\varepsilon_{i, t}
$$

Donde:

$G I N I_{i, t}=$ índice de Gini de la región i al final del período t

$\alpha=$ término constante

$\beta=$ coeficiente de la variable predictora

8 Forbes (2000) estudia el efecto de la desigualdad del ingreso sobre el crecimiento, se sigue su metodología de análisis de datos de panel, adaptada a este estudio. 
$B=$ vector de coeficientes de las variables de control

$I_{N G R S O}{ }_{i, t-1}=$ medida de ingreso de la región i al comienzo del período $\mathrm{t}$ (final del período $\mathrm{t}-1)$.

$X_{i, t-1}=$ vector de variables de control

$\varepsilon=$ término de error.

La Tabla 1 presenta los mejores resultados obtenidos para distintas combinaciones de las variables regresoras (ver Anexo A con la estadística descriptiva de las principales variables utilizadas, el Anexo B con el detalle de los índices de Gini por región y año, y el Anexo E con las definiciones de las variables).

El modelo I, siguiendo la primera estimación realizada en el estudio de Barro (2000), busca indagar más en el resultado del Gráfico 1, por lo que utiliza como variables explicativas el $L N$ (PIB per cápita) y su cuadrado como únicos regresores (además del término constante). Ambas variables son significativas, demostrando que efectivamente, en las regiones chilenas, el PIB per cápita tiene un efecto negativo sobre la desigualdad, es decir, que regiones con mayor PIB per cápita, tienden a reducir las desigualdades. Sin embargo, esta relación no se mantiene, llega un punto en que mayores ingresos, incrementan las desigualdades, sobretodo, cuando los incrementos provienen de la población más rica. Este primer modelo, probablemente contiene variables omitidas y, por tanto, los resultados pudieran estar eventualmente sesgados. Para minimizar lo anterior se prueba utilizar efectos fijos (modelo II). Si bien mejora el ajuste del modelo $\left(\mathrm{R}^{2}=0,404\right)$, las variables explicativas no son significativas.

Los modelos siguientes incorporan las variables explicativas escogidas de acuerdo a la literatura. Se utiliza MCO porque se demostró, a través de una Prueba F restrictiva, que es mejor utilizar MCO que un modelo de Efectos Fijos (excepto en la columna 1). La hipótesis nula es que todas las variables dicotómicas regionales son iguales a 0. Al no rechazar esta hipótesis, se dice que es mejor utilizar los MCO.

La tercera columna incorpora tres nuevos factores. Se demuestra que las regiones más desiguales inicialmente, tienden a ser también las regiones más desiguales al final del período. Se concluyó en Mieres (2019) que las regiones de Chile con mayor índice de Gini inicial tendían a reducir más la desigualdad a lo largo del tiempo (convergencia), sin embargo, el modelo III demuestra que la desigualdad es un fenómeno persistente en el tiempo. Cuando una región arrastra un cierto nivel de desigualdad del ingreso, la desigualdad tiende a permanecer y el mercado por sí solo no es capaz de corregirla. Es por ello que las políticas redistributivas han de cobrar protagonismo si la desigualdad social es una prioridad. En Chile, la redistribución, a través del gasto social, forma parte importante del ingreso de las familias más vulnerables ${ }^{9}$, por tanto, es esencial

9 De acuerdo con Sunkel e Infante (2009), en 2006, para el 40\% de la población de menores ingresos, solo un $40,5 \%$ de su rentas provenía del trabajo, un mayoritario 50,5\% provenía del gasto social. 


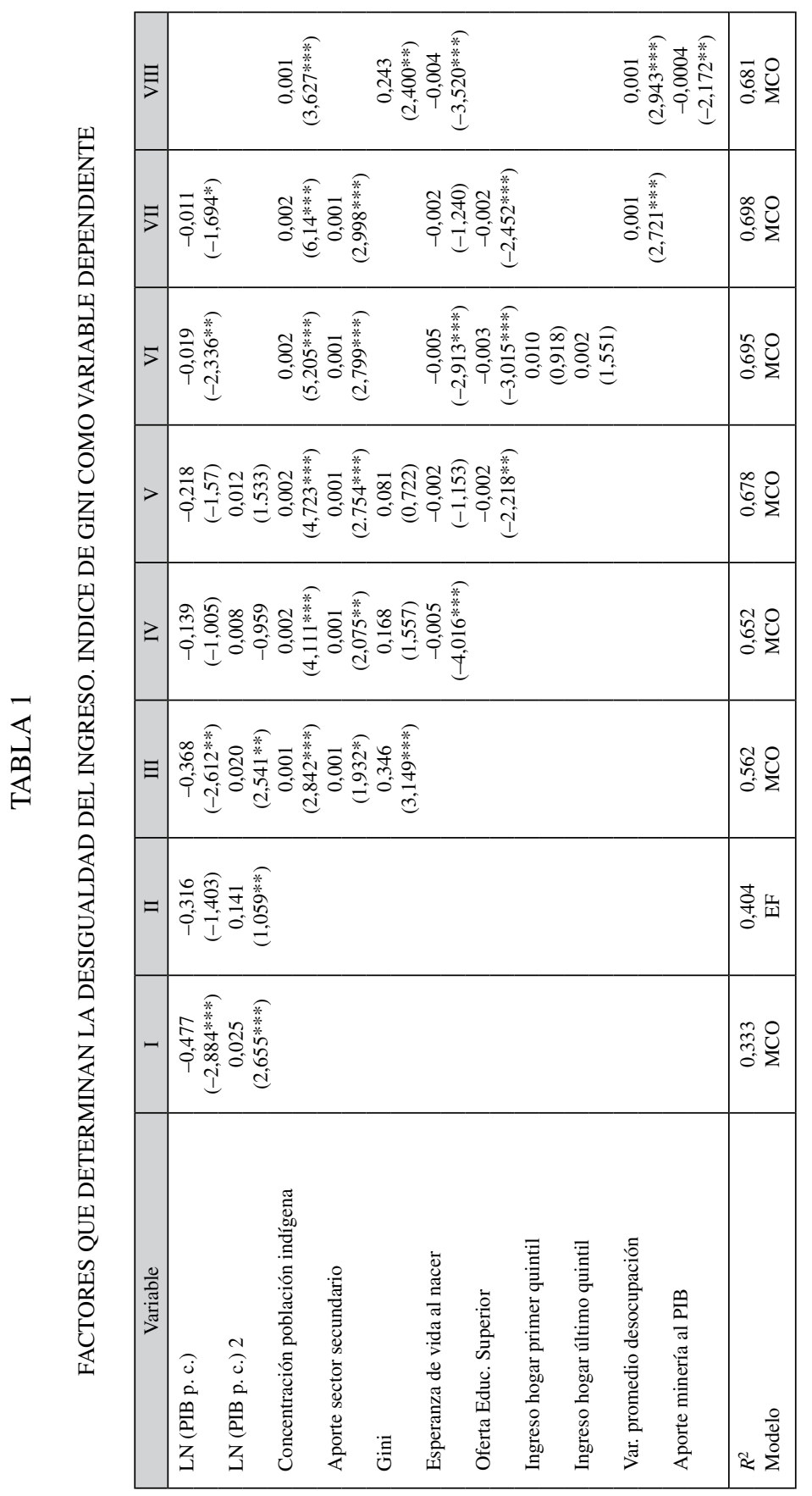

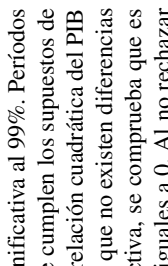

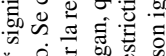
*

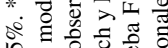

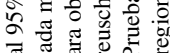

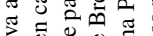

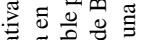
记 .

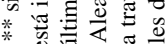

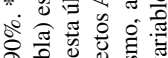

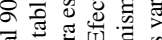

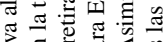

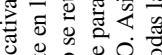
包苟

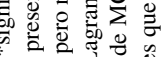

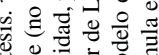
象

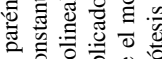

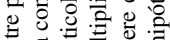

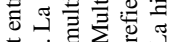

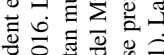
至 虫它总

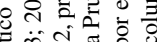

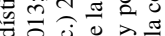

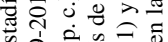

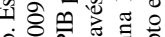

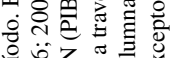
응 范 西

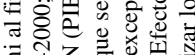

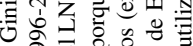

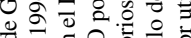

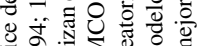

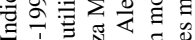

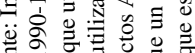
ब

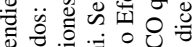

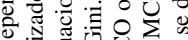

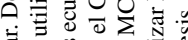

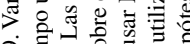
oิ

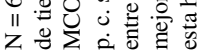


para la reducción de la pobreza, sin embargo, no han sido suficientemente efectivas ante la alta inequidad. Para reducir la resistente desigualdad del ingreso en el país, son necesarias más políticas distributivas que se preocupen a priori de la generación del ingreso (Sunkel e Infante, 2009).

Otras variables con efecto positivo sobre la desigualdad del ingreso son, la concentración de población indígena y el aporte del sector secundario al PIB. La desigualdad tiende a ser más alta en regiones con mayor concentración de población indígena, respecto del total nacional. Por su parte, se observa que regiones con mayor aporte del sector secundario, ceteris paribus, tienden a ser menos equitativas, posiblemente porque los beneficios captados en este rubro no son inclusivos con toda la comunidad.

El modelo IV adiciona una variable social, la esperanza de vida al nacer, mejorando el $R^{2}$ en un $9 \%$ respecto al modelo anterior, sin embargo, tanto el $L N$ $(P I B$ per cápita $)$ como el $L N(P I B p c)^{2}$ dejan de ser significativos, al igual que el Gini inicial. Esto puede ser debido a que la esperanza de vida al nacer es una variable muy correlacionada con el PIB per cápita (correlación positiva y significativa al 99\%) y con otras variables omitidas, también correlacionadas con el PIB per cápita y la desigualdad, por ejemplo la atención sanitaria. Se aprecia que aquellas regiones con mejor nivel de salud, expuesto a través de la esperanza de vida, tienden a reducir las desigualdades.

El modelo V incorpora la variable oferta de educación superior como medida de capital humano calificado, mejorando el modelo. La variable tiene una relación negativa y significativa con el Gini. Regiones con mayor oferta educativa por habitante, tienden a tener menor desigualdad del ingreso. Esta variable es robusta, ya que se mantiene significativa y conserva el signo en los distintos modelos donde se utiliza. El Gini inicial sigue sin ser significativo, posiblemente por la alta correlación entre ambas variables (correlación negativa y significativa al 99\%). Acorde a lo anterior, los modelos que siguen utilizan una variable o la otra, dependiendo cual permite un mayor ajuste.

El modelo VI incorpora, al igual que el estudio de Solimano y Torche (2008), el ingreso autónomo del hogar. Se utilizan los quintiles del ingreso autónomo en lugar del Gini como controles de la desigualdad inicial y se mantiene el PIB per cápita. Este modelo se ajusta en un 69,5\%. Tanto la concentración de población indígena como el aporte del sector secundario y la oferta de educación superior siguen siendo relevantes y el PIB per cápita vuelve a ser significativo. En este caso ni el ingreso del $20 \%$ más pobre de la población ni el ingreso del $20 \%$ más rico son variables significativas en el modelo.

El modelo VII es el de mayor ajuste, con un $R^{2}$ de casi $70 \%$. Se excluyen los ingresos del primer y último quintil e incorpora la variable variación del desempleo. Se demuestra que la variable tiene un efecto positivo y significativo sobre la desigualdad 
del ingreso. El desempleo produce una merma de ingresos en la población desocupada y por tanto provoca más desigualdad, dado que la desocupación suele concentrarse más en los quintiles de más bajos ingresos y poca cualificación (ver por ejemplo Jiménez y Ruedi (1998)), por tanto, fomentar la educación y la formación contribuirá a disminuir las tasas de desempleo, a aumentar los ingresos de las familias más pobres y en consecuencia reducir la desigualdad de la renta.

La última columna, considera el aporte de la minería en lugar del sector secundario y excluye el PIB per cápita. Esta variable esta muy correlacionada con el PIB per cápita, ya que, en Chile la actividad minera es una parte primordial de la productividad del país. El efecto de esta variable es el opuesto al provocado por el sector secundario. Las regiones con mayor aporte de la minería tienden a ser más equitativas en la renta ${ }^{10}$.

Igualmente, se probó incorporar como variable de control la tasa de crecimiento promedio del PIB per cápita (aunque no se presenta en la tabla), el coeficiente de esta variable es positivo y únicamente significativo cuando se controla por la concentración de población indígena y el aporte del sector secundario. Se infiere que períodos de alto crecimiento económico tienden a aumentar la participación de la población de mayores ingresos, a expensas del resto de la población. Recordando a Merchand (2017), este señala que las desigualdades entre regiones pueden mantenerse en el tiempo e incluso crecer junto con la economía de un país, ya que existe un centro que aprovecha los mayores beneficios del crecimiento, mientras que las regiones de la periferia pierden esa oportunidad.

Los resultados exponen la relevancia de la presencia de población indígena en las regiones. Las desigualdades tienden a ser mayores en aquellas regiones con mayor concentración de población perteneciente a una etnia, respecto al total nacional. Lo anterior enfatiza la desventaja económica de la población indígena, además el resultado es robusto, ya que el coeficiente varia entre 0,001 y 0,002 en las distintas ecuaciones. De acuerdo con la CASEN, en 2015, el ingreso promedio de los hogares cuyo jefe de hogar pertenecía a alguna etnia fue un $43 \%$ más bajo que el de un hogar cuyo jefe de hogar se declara no indígena. Asimismo, el 18,3\% se encontraba en situación de pobreza. Cada año existe una diferencia significativa entre la tasa de desempleo de la población indígena y no indígena, siendo en 2015 de un 8,3\% para los primeros y un menor 7,4\% para los segundos. A nivel educacional, la población indígena también se encuentra más vulnerable, principalmente las personas mayores. El 16,8\% de los mayores de 60 años se declaran analfabetos y un 46,6\% de los mayores de 19 años

10 Esta ecuación también se probó utilizando la oferta de educación superior en vez del Gini inicial, la variable resultó igualmente significativa y negativa, siendo el ajuste del modelo de un 67,7\%. 
no han alcanzado la enseñanza media completa ( $38 \%$ de la población no indígena) ${ }^{11}$. De acuerdo con Todaro y Smith (2012), aunque es difícil obtener información de los países respecto a población indígena, la evidencia empírica en Latinoamérica demuestra que la mayoría de los grupos indígenas viven en la pobreza extrema y que ser indígena incrementa las probabilidades de que una persona tenga mala salud, esté desnutrida, sea analfabeta y esté desempleada.

Este trabajo demuestra que el sector económico más potente, en el sentido del aporte al PIB regional, juega un papel importante en la reducción de la desigualdad, pero el efecto negativo provocado por el sector secundario parece haber tenido una influencia mayor. Se observa que la desigualdad del ingreso tiende a aumentar en regiones intensivas en el sector secundario (manufactura; electricidad, agua y gas y sector construcción), mientras que las regiones intensivas en minería tienden a ser más equitativas. Se infiere que en estas regiones, las riquezas provenientes de la minería, son repartidas más inclusivamente entre la población trabajadora. De acuerdo con Macías (2014, pp. 119) "un crecimiento equilibrado de la productividad entre la agricultura y la industria genera menos desigualdad que un crecimiento de la productividad sesgado hacia la industria", haciendo referencia a las diferencias campo-ciudad que puede provocar una mayor acumulación de capital físico en la industria ${ }^{12}$.

La situación de la minería es distinta, a pesar de ser un sector que al igual que la industria acumula capital físico. Entonces ¿por qué las regiones intensivas en minería son más equitativas? Una de las posibles respuestas es que la minería tiene una alta demanda de mano de obra no calificada, por tanto, los salarios de estos trabajadores son relativamente altos, comparados con los del sector industrial ${ }^{13}$. Por otro lado, de acuerdo con el estudio de Betancour y Maldonado (2013), la minería estaba entre los tres sectores económicos con mayor variación de la tasa de empleo. "En las cuatro regiones mineras se producen los mayores crecimientos de personas ocupadas en el país entre 1986 y 2012, con un incremento que fluctúa entre $150 \%$ y $117 \%$, con un promedio de $139 \%$. Esto determina que la participación de estas regiones en el total del empleo del país crece desde 10\% a 12\%" (Betancour y Maldonado, 2013, pp. 115). El desempleo por sector económico también es relevante, de acuerdo al INE, en el último semestre 2015 el sector secundario tenía las mayores tasas de cesantía, sobre todo en el sector construcción.

Otras variables, como los años de escolaridad, no se han mostrado significativas, a pesar de tener una correlación alta y negativa con la variable dependiente. Probablemente,

11 Puede ver el informe completo en Pueblos indígenas, síntesis de resultados del Ministerio de Desarrollo Social (2017).

12 De acuerdo con Macías (2014), el crecimiento que surge por la acumulación de capital físico puede aumentar los niveles de desigualdad, sobretodo en países pobres o de ingresos medios, al aumentar las disparidades entre el campo y la ciudad.

13 No se puede ahondar en este tema, ya que este estudio no trabaja con salarios medios relativos. Sin embargo, es muy interesante tenerlo en cuenta para futuras investigaciones. 
esto se debe a su alta correlación con las medidas de ingreso y ocupación. Igualmente se utilizó la variable becas de postgrado (no se presenta en la tabla) con base en la información entregada por CONICYT respecto a becas nacionales de magíster y doctorado. Si bien la relación entre esta variable y el Gini era también negativa, no era significativa al incorporarse en los distintos modelos. Finalmente, la variable oferta de educación superior ha resultado significativa y robusta como medida de capital humano calificado. Se puede inferir que el esfuerzo de las políticas de acceso a la educación superior, sobre todo aquellas implementadas en los últimos 13 años, han sido importantes para combatir la desigualdad ${ }^{14}$.

Hasta ahora se han realizado las estimaciones a través del modelo de MCO, ya que en las ecuaciones de la Tabla 1 (excepto la ecuación I), el efecto individual de las regiones no ha sido significativo (ver pie de la Tabla 1). A continuación se evalúa si existe un efecto específico temporal, incorporando variables dummy de períodos. Se estima el modelo de Efectos Fijos Temporales, suponiendo que las diferencias entre los distintos períodos son constantes (o fijas), por ello se debe estimar cada intercepto. Una manera para permitir que el intercepto varíe con respecto a cada período es la técnica de "las variables dicotómicas de intersección diferencial" (Aparicio y Márquez, 2005), que se presenta de la siguiente forma:

$$
\operatorname{GINI}_{i, t}=v_{t}+\beta_{1} \operatorname{INGRESO}_{i, t-1}+B X_{i, t-1}+\varepsilon_{i, t}
$$

Donde $v_{t}$ es un vector de variables dicotómicas para cada período. Como forma de averiguar si existen efectos fijos en el tiempo se utiliza una prueba $F$ restrictiva. La hipótesis nula es que todas las variables dicotómicas son iguales a 0 . Si se rechaza la prueba, significa que al menos algunas variables dicotómicas pertenecen al modelo, y por lo tanto es necesario utilizar los Efectos Fijos. Los resultados de la prueba $F$ indican que para todas las ecuaciones de la Tabla 1 existen Efectos Fijos en el tiempo. Estos resultados se muestran en la segunda tabla.

Se observa en la Tabla 2, que la variable tiempo mejora el ajuste del modelo en todos los casos, destacan los modelos X, XI y XII. De la segunda columna se extrae que el efecto del año 2000 sobre la desigualdad del ingreso fue 0,038 puntos superior, en promedio, al del período base (1994). En los últimos dos períodos, el efecto sobre el Gini fue significativamente menor que el del período base. Se comprueba la robustez de la variable concentración de población indígena, la cual continua siendo positiva y significativa en cada una de las ecuaciones estimadas.

14 Por ejemplo, en el año 2007 se crea el Consejo Asesor Presidencial para la Calidad de la Educación, comisión convocada por la expresidenta Michelle Bachelet para el estudio de reformas a la Educación en Chile. Fue creada como consecuencia de las movilizaciones estudiantiles que se realizaron en el país entre mayo y junio de 2006. 


\section{TABLA 2}

\section{FACTORES QUE DETERMINAN LA DESIGUALDAD DEL INGRESO. \\ MCO CON EFECTOS FIJOS EN EL TIEMPO. INDICE DE GINI COMO VARIABLE DEPENDIENTE}

\begin{tabular}{|c|c|c|c|c|c|c|}
\hline Variable & IX & $\mathrm{X}$ & $\mathrm{XI}$ & XII & XIII & XIV \\
\hline LN (PIB p. c.) & $\begin{array}{c}-0,410 \\
(-2,327 * *)\end{array}$ & $\begin{array}{c}-0,261 \\
\left(-2,011^{* *}\right)\end{array}$ & $\begin{array}{c}-0,255 \\
(-1,863 *)\end{array}$ & $\begin{array}{c}-0,028 \\
(-3,431 * * *)\end{array}$ & $\begin{array}{c}-0,016 \\
(-2,513 * *)\end{array}$ & \\
\hline LN (PIB p. c.) 2 & $\begin{array}{c}0,021 \\
(2,132 * *)\end{array}$ & $\begin{array}{c}0,014 \\
\left(1,931^{*}\right)\end{array}$ & $\begin{array}{c}0,014 \\
(1,778 *)\end{array}$ & & & \\
\hline Concentración población indígena & & $\begin{array}{c}0,002 \\
(4,580 * * *)\end{array}$ & $\begin{array}{c}0,002 \\
(4,220 * * *)\end{array}$ & $\begin{array}{c}0,002 \\
(4,627 * * *)\end{array}$ & $\begin{array}{c}0,002 \\
(5,444 * * *)\end{array}$ & $\begin{array}{c}0,001 \\
(4,174 * * *)\end{array}$ \\
\hline Aporte sector secundario & & $\begin{array}{c}0,001 \\
(1,896 *)\end{array}$ & $\begin{array}{c}0,001 \\
(1,597)\end{array}$ & $\begin{array}{c}0,001 \\
(-1,450)\end{array}$ & $\begin{array}{c}0,001 \\
(-1,279)\end{array}$ & \\
\hline Gini & & $\begin{array}{c}0,142 \\
(1,402)\end{array}$ & $\begin{array}{c}0,145 \\
(1,393)\end{array}$ & & & $\begin{array}{c}0,157 \\
(1,513)\end{array}$ \\
\hline Esperanza de vida al nacer & & $\begin{array}{c}-0,002 \\
(-0,617)\end{array}$ & $\begin{array}{c}-0,002 \\
(-0,616)\end{array}$ & $\begin{array}{c}-0,006 \\
\left(-1,865^{*}\right)\end{array}$ & $\begin{array}{c}-0,006 \\
(-1,797 *)\end{array}$ & $\begin{array}{c}-0,006 \\
(-2,207 * *)\end{array}$ \\
\hline Oferta Educ. Superior & & & $\begin{array}{l}0,0002 \\
(0,149)\end{array}$ & $\begin{array}{l}0,0001 \\
(0,067)\end{array}$ & $\begin{array}{c}0,0004 \\
(0,36)\end{array}$ & \\
\hline Ingreso hogar primer quintil & & & & $\begin{array}{c}0,009 \\
(0,782)\end{array}$ & & \\
\hline Ingreso hogar último quintil & & & & $\begin{array}{c}0,002 \\
\left(1,870^{*}\right)\end{array}$ & & \\
\hline Var. promedio desocupación & & & & & $\begin{array}{l}0,0003 \\
(0,718)\end{array}$ & $\begin{array}{l}0,0004 \\
(0,994)\end{array}$ \\
\hline Aporte minería al PIB & & & & & & $\begin{array}{c}-0,0004 \\
(-2,222 * *)\end{array}$ \\
\hline $1996-2000$ & $\begin{array}{c}0,041 \\
(3,121 * * *)\end{array}$ & $\begin{array}{c}0,038 \\
(3,183 * * *)\end{array}$ & $\begin{array}{c}0,038 \\
(3,018 * * *)\end{array}$ & $\begin{array}{c}0,032 \\
(2,622 * *)\end{array}$ & $\begin{array}{c}0,036 \\
(2,422 * *)\end{array}$ & $\begin{array}{c}0,032 \\
(2,381 * *)\end{array}$ \\
\hline $2003-2006$ & $\begin{array}{c}0,007 \\
(0,418)\end{array}$ & $\begin{array}{c}0,010 \\
(0,375)\end{array}$ & $\begin{array}{c}0,012 \\
(0,397)\end{array}$ & $\begin{array}{c}0,015 \\
(0,552)\end{array}$ & $\begin{array}{c}0,034 \\
(1,230)\end{array}$ & $\begin{array}{c}0,037 \\
(1,517)\end{array}$ \\
\hline 2009-2013 & $\begin{array}{c}-0,019 \\
(-9,940)\end{array}$ & $\begin{array}{c}-0,011 \\
(-0,374)\end{array}$ & $\begin{array}{c}-0,011 \\
(-0,365)\end{array}$ & $\begin{array}{c}-0,018 \\
(-0,648)\end{array}$ & $\begin{array}{c}0,010 \\
(0,337)\end{array}$ & $\begin{array}{l}0,015 \\
(0,57)\end{array}$ \\
\hline $2013-2016$ & $\begin{array}{c}-0,014 \\
(-0,565)\end{array}$ & $\begin{array}{c}-0,004 \\
(-0,119)\end{array}$ & $\begin{array}{l}-0,004 \\
(-0,12)\end{array}$ & $\begin{array}{c}-0,020 \\
(-0,645)\end{array}$ & $\begin{array}{c}0,010 \\
(0,327)\end{array}$ & $\begin{array}{c}0,018 \\
(0,626)\end{array}$ \\
\hline$R^{2}$ & 0,697 & 0,764 & 0,764 & 0,776 & 0,744 & 0,747 \\
\hline
\end{tabular}

$\mathrm{N}=69$. Var. Dependiente: Indice de Gini al final del período. Estadístico T-student entre paréntesis. *significativa al 90\%. ** significativa al 95\%.*** significativa al 99\%. Estimación de MCO con dummy (período de tiempo). La constante (no presente en la tabla) está incluida en cada modelo. Modelo IX modelo con efectos fijos por región y en el tiempo.

El período 1996-2000 destaca entre todos los períodos al presentar el mayor coeficiente, positivo y significativo, en todos los modelos. La época de dictadura militar en Chile se caracterizó por una gran apertura comercial y financiera (unilateral), una reducción del papel Estatal (aumentando la privatización de empresas del Estado) y un uso extensivo de los mecanismos de mercado (Sunkel e Infante, 2009), propiciando la desigualdad del ingreso. Entre los años 1990 y 2000, Chile se caracterizó por un gran dinamismo económico, producto de la vuelta a la democracia suscitada en 
1989. En este período los ingresos promedio se incrementaron, principalmente en los grupos con mayores ingresos, lo que se tradujo en un aumento de la desigualdad (sin embargo, el nivel de desigualdad no fue superior al vivido en época de dictadura). Particularmente, entre 1998-2003 la distribución de la renta experimentó un leve deterioro como resultado de las políticas de ajuste suscitadas por la crisis asiática (Sunkel e Infante, 2009). Las décadas siguientes tuvieron una menor dinámica y aquí fueron los quintiles de menores ingresos los que más aumentaron proporcionalmente.

Durante los años 2000-2015, los ingresos del hogar del primer quintil aumentaron $165 \%$, mientras que los del último quintil lo hicieron en un $71 \%$ (analizando cada período estudiado, por separado, se mantiene la tendencia, ver Anexo C). En consecuencia, la desigualdad cae respecto al año base (como se observa en la Tabla 2). Es claro que períodos en los que el ingreso de los ricos aumenta proporcionalmente más que el de los pobres, son épocas donde aumenta la desigualdad, mientras que períodos donde el ingreso de las familias más pobres crecen proporcionalmente más, las desigualdades caen. De acuerdo con Contreras (1999) y Sunkel (2009), en Chile los altos niveles de desigualdad se explican por el comportamiento de la población de mayores ingresos. Este último autor lo atribuye a la alta capacidad de la población más rica de mantener o incluso aumentar su participación en el ingreso total, versus la menor capacidad de la población de menores ingresos.

Se aprecia en el modelo XII, que ahora la variable ingreso último quintil es significativa y positiva, reforzando lo dicho en el párrafo anterior. Esto podría parecer obvio, sin embargo no lo es, ya que de acuerdo a la teoría del "goteo" ("trickle down"), el favorecer a los estratos más ricos de la población, aquellos con mayor capacidad de acumulación de capital, tiende a favorecer al resto de la sociedad, a través de un goteo que llega a las personas menos favorecidas, en consecuencia la desigualdad se reduciría. Esta teoría, que se fundamenta en la hipótesis de Kuznets, no se cumple en este caso (al menos en el período de tiempo estudiado). Varios autores, como Arndt (1983), Guillén (2004) y el más señalado en el último tiempo, Piketty (2014), han revelado esta teoría como falsa.

Los últimos dos períodos se caracterizaron por un efecto menor sobre la desigualdad del ingreso (respecto al período base). Como se mencionó anteriormente, la vuelta a la democracia fue necesaria para poder implementar políticas redistributivas, muchas de las cuales (como las referentes al acceso a educación) tienen efectos en el largo plazo, razón por la cual, en los primeros años de democracia (con los Gobiernos de la concertación) podemos ver aún altos niveles de desigualdad respecto al año base. Sin embargo, se puede pensar, que los efectos negativos de los últimos períodos son consecuencia de los actos y políticas aplicadas desde los años 90.

La relación cuadrática (parábola) del Gráfico 2, muestra el ajuste del modelo XII para la relación entre LN (PIB per cápita) inicial y el Gini final. Es decir, una vez descontado el efecto del período, los ingresos del primer y último quintil, la concentración de población indígena, el aporte del sector secundario, la esperanza de vida al nacer y la oferta de educación superior sobre la desigualdad del ingreso. La 
pendiente negativa viene determinada, por tanto, por el coeficiente de la variable PIB per cápita. Si bien el modelo tiene un ajuste relativamente alto $(77,6 \%)$, todavía existe cierta dispersión al rededor de los datos, posiblemente por alguna variable omitida (por ejemplo el nivel de apertura económica ${ }^{15}$ ). El estudio de Barro (2000), que utiliza la relación de exportaciones más importaciones respecto al PIB, como variable que representa el nivel de apertura económica, concluye que el efecto de este factor sobre la desigualdad del ingreso depende del nivel de desarrollo de los países. Y sugiere que una mayor apertura internacional aumentaría la desigualdad en los países ricos y la reduciría en los países pobres. Lo anterior, ya que países que están relativamente con alta dotación de mano de obra no calificada (países de menores ingresos), tienden a importar más productos intensivos en capital humano y físico, y exportar productos intensivos en mano de obra no calificada, por tanto, una mayor apertura tendería a elevar los salarios relativos de la mano de obra no calificada, repercutiendo en una menor desigualdad de ingresos.

\section{GRAFICO 2}

LN (PIB PER CAPITA INICIAL) V/S INDICE DE GINI (PARTE NO EXPLICADA), DE ACUERDO AL MODELO XII DE LA TABLA 2

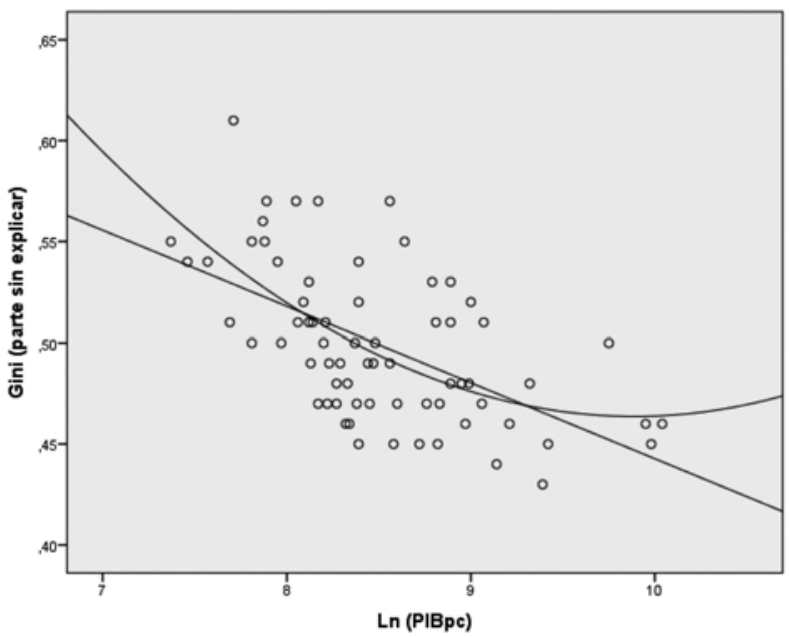

15 El Servicio Nacional de Aduanas informa que existen datos de exportaciones por regiones solo a partir del año 2002. 
Finalmente, para verificar la robustez de los resultados, se realiza una regresión utilizando como variable dependiente una medida de desigualdad diferente, el ratio 20/20 (en función logarítmica). La Tabla 3 muestra estas estimaciones. De la primera columna se extrae que el PIB per cápita es significativo al 90\%, manteniendo el signo negativo de los modelos anteriores. Asimismo, se observa que el ingreso del $20 \%$ más pobre es significativo (al 95\% de confianza) para explicar las diferencias entre el quintil más rico y el más pobre. A partir del modelo XV, al agregar nuevas variables, tanto el PIB per cápita como los ingresos por quintil dejan de ser significativos.

Utilizando esta nueva medida de desigualdad existe un mayor ajuste del modelo (siendo el $R^{2}$ igual a $81 \%$ y $82 \%$ en los modelos XVIII y XIX respectivamente) y el valor de los coeficientes de los distintos estimadores se incrementa. Por ejemplo, el coeficiente del factor concentración de la población indígena varía entre 0,01 y 0,013 en las distintas ecuaciones.

La Tabla 3 refuerza la importancia del sector económico (minería y sector secundario), la concentración de la población indígena y el capital humano (tanto la educación como esperanza de vida), como factores determinantes de la desigualdad del ingreso. Estas variables tienen el mismo efecto sobre la desigualdad, independientemente si esta se mide a través del Gini o del ratio 20/20, y son significativos siempre, excepto la esperanza de vida al nacer en la ecuación XVIII. Al utilizar el ratio 20/20, el PIB per cápita pierde significancia, sin embargo, los años de escolaridad son ahora un factor significativo ${ }^{16}$. En cada caso se observa que las regiones con mayor nivel educacional tienden a ser menos desiguales. Estos resultados invitan a redoblar los esfuerzos para reducir la desigualdad de oportunidades y fomentar un sistema educativo que logre llegar a los estratos más pobres de la población, sobretodo a la educación superior. "La equiparación de oportunidades no implica por sí misma una reducción del nivel de desigualdad ex-post, pero tiende a que las diferencias de ingreso respondan más a factores personales (esfuerzo y habilidades), que a diferencias en el capital inicial de los individuos y de las familias" (Gasparini, Marchionni y Sosa, 2001, pp. 30).

16 Al igual que en las Tablas 1 y 2, se prueban los modelos utilizando la variable oferta de educación superior (en vez de años de escolaridad), en este caso la variable es significativa en las ecuaciones XVI y XVII incrementando muy poco el ajuste del modelo. Al utilizar esta variable, la esperanza de vida pierde significancia. 
TABLA 3

FACTORES QUE DETERMINAN LA DESIGUALDAD DEL INGRESO. RATIO 20/20 COMO VARIABLE DEPENDIENTE

\begin{tabular}{|c|c|c|c|c|c|}
\hline Variable & $\mathrm{XV}$ & XVI & XVII & XVIII & XIX \\
\hline LN (PIB p. c.) & $\begin{array}{c}-0,121 \\
\left(-1,972^{*}\right)\end{array}$ & $\begin{array}{c}0,143 \\
(1,417)\end{array}$ & $\begin{array}{c}0,004 \\
(0,067)\end{array}$ & $\begin{array}{l}-0,076 \\
(-1,405)\end{array}$ & $\begin{array}{c}-0,165 \\
\left(-3,013^{* * *}\right)\end{array}$ \\
\hline Ingreso hogar primer quintil & $\begin{array}{c}-0,188 \\
(-2,642 * *)\end{array}$ & $\begin{array}{l}-0,057 \\
(-0,967)\end{array}$ & $\begin{array}{l}-0,050 \\
(-0,859)\end{array}$ & $\begin{array}{c}0,012 \\
-0,203\end{array}$ & $\begin{array}{c}0,020 \\
(0,326)\end{array}$ \\
\hline Ingreso hogar último quintil & $\begin{array}{c}0,008 \\
(1,230)\end{array}$ & $\begin{array}{c}0,002 \\
(0,247)\end{array}$ & $\begin{array}{c}0,007 \\
(1,065)\end{array}$ & $\begin{array}{c}0,005 \\
(1,000)\end{array}$ & $\begin{array}{c}0,008 \\
(1,430)\end{array}$ \\
\hline Concentración población indígena & & $\begin{array}{c}0,012 \\
\left(4,909^{* * *}\right)\end{array}$ & $\begin{array}{c}0,013 \\
(5,441 * * *)\end{array}$ & $\begin{array}{c}0,012 \\
\left(6,430^{* * *}\right)\end{array}$ & $\begin{array}{c}0,010 \\
\left(5,110^{* * *}\right)\end{array}$ \\
\hline Esperanza de vida al nacer & & $\begin{array}{c}-0,020 \\
\left(-2,281^{* *}\right)\end{array}$ & $\begin{array}{c}-0,020 \\
\left(-2,314^{* *}\right)\end{array}$ & $\begin{array}{c}-0,022 \\
(-1,132)\end{array}$ & $\begin{array}{c}-0,040 \\
(-2,457 * *)\end{array}$ \\
\hline Años de escolaridad & & $\begin{array}{c}-0,094 \\
\left(-2,303^{* *}\right)\end{array}$ & $\begin{array}{c}-0,082 \\
\left(-2,110^{* *}\right)\end{array}$ & $\begin{array}{c}-0,061 \\
\left(-1,762^{*}\right)\end{array}$ & $\begin{array}{c}-0,117 \\
(-2,521 * *)\end{array}$ \\
\hline Aporte minería al PIB & & $\begin{array}{c}-0,005 \\
\left(-2,197^{* *}\right)\end{array}$ & & & \\
\hline Aporte sector secundario & & & $\begin{array}{c}0,006 \\
(2,691 * * *)\end{array}$ & $\begin{array}{c}0,005 \\
\left(2,176^{* *}\right)\end{array}$ & \\
\hline Aporte s. silvo-agropecuario al PIB & & & & & $\begin{array}{c}-0,010 \\
\left(-2,471^{* *}\right)\end{array}$ \\
\hline $1996-2000$ & & & & $\begin{array}{c}0,281 \\
(4,041 * * *)\end{array}$ & $\begin{array}{c}0,358 \\
\left(5,779^{* * *}\right)\end{array}$ \\
\hline 2003-2006 & & & & $\begin{array}{c}0,168 \\
(1,051)\end{array}$ & $\begin{array}{c}0,315 \\
(2,396 * *)\end{array}$ \\
\hline 2009-2013 & & & & $\begin{array}{c}0,041 \\
(0,238)\end{array}$ & $\begin{array}{c}0,178 \\
(1,219)\end{array}$ \\
\hline 2013-2016 & & & & $\begin{array}{c}0,032 \\
(0,168)\end{array}$ & $\begin{array}{c}0,211 \\
(1,332)\end{array}$ \\
\hline$R^{2}$ & 0,381 & 0,653 & 0,666 & 0,811 & 0,816 \\
\hline
\end{tabular}

$\mathrm{N}=69$. Var. Dependiente: LN (ratio 20/20). Estadístico T-student entre paréntesis. *significativa al 90\%. ** significativa al 95\%.*** significativa al 99\%. Estimación de MCO con dummy (período de tiempo) en la última ecuación. La constante (no presente en la tabla) está incluida en cada modelo. Se utiliza MCO porque se demostró, a través de la Prueba del Multiplicador de Lagrange para Efectos Aleatorios de Breusch y Pagan, que no existen diferencias entre usar MCO o Efectos Aleatorios (excepto en la columna 1) y por ende se prefiere el modelo de MCO. Asimismo, a través de una Prueba $F$ restrictiva, se comprueba que es mejor utilizar MCO que un modelo de Efectos Fijos (excepto en la columna 1). La hipótesis nula es que todas las variables dicotómicas regionales son iguales a 0 . Al no rechazar esta hipótesis, se dice que es mejor utilizar los MCO. La ecuación XIV probablemente contiene variables omitidas y, por tanto, los resultados pueden estar sesgados. Al utilizar EF para minimizar el sesgo, el ajuste del modelo no mejora y las variables explicativas no son significativas. 
Igualmente, se observan efectos fijos de período, siendo el período 1996-2000 significativo en los modelos XVIII y XIX, resultado consistente con los obtenidos previamente. Al sustituir el aporte del sector secundario del modelo XVII, por el factor aporte del sector silvoagropecuario (modelo XIX), este es también significativo, demostrando que las regiones con mayor aporte de este sector al PIB regional, tienden a disminuir las desigualdades. Esto se puede explicar, ya que la población que trabaja en el sector silvoagropecuario es mayormente de bajos ingresos, por ende, mayores rentas en esta área van en directo beneficio del quintil más pobre, reduciendo las brechas entre el $20 \%$ más rico y más pobre. Los resultados del estudio de Bourguignon y Morrisson (1998) sugieren al respecto que, en muchos países, incrementar el nivel de productividad del sector agrícola podría ser una de las formas más eficientes de reducir la pobreza y la desigualdad. En esta última columna, tanto el PIB per cápita como la esperanza de vida al nacer, vuelven a ser significativos.

\section{CONCLUSIONES Y LIMITACIONES}

La construcción de una amplia base de datos regional en Mieres (2019) permitió dar una mirada a la dinámica de la desigualdad en Chile, retratando las diferencias regionales. Lo que dio paso al presente análisis empírico enfocado en el período 1990-2016 y que exploró los principales determinantes de la desigualdad del ingreso en Chile.

El análisis econométrico siguió la metodología utilizada en los estudios de Forbes (2000) y Barro (2000) y conjuga las variables que, de acuerdo a Kuznets (1955), Barro (2000) y otros autores de reconocido prestigio, influyen sobre la desigualdad del ingreso, siendo la variable dependiente el índice de Gini. Los resultados revelaron una relación cuadrática entre el PIB per cápita y el Gini, pero no como lo plantea Kuznets, sino más bien a la inversa. Al principio, los altos ingresos disminuyen las desigualdades, lo cual es un proceso deseable para un crecimiento económico inclusivo. Sin embargo, la tendencia negativa se convierte en positiva a partir de un cierto nivel de renta, alertando que la actual modalidad de crecimiento en Chile exigiría sacrificar la equidad en aras de obtener una mayor eficiencia. Esto es relevante para la política pública, la cual debe incentivar todas aquellas fuerzas que reducen la desigualdad, y de esta manera, cambiar la forma cuadrática de la relación entre renta y desigualdad. Esta relación se observa también en países como Estados Unidos, Reino Unido, Costa Rica e India.

Lo que marca la desigualdad en Chile es su aún elevado nivel y su persistencia, ya que, a pesar de existir convergencia, el Gini al comienzo de un período tiene un efecto positivo sobre el Gini al final de este. Los resultados indican que el sector económico más potente, en el sentido del aporte al PIB regional, es muy importante para determinar las desigualdades en Chile, destacando los sectores secundario y minería. Las regiones intensivas en el sector secundario tienden a aumentar las 
desigualdades, mientras que lo contrario sucede en las regiones con mayor aporte del sector minero. Se infiere que en estas regiones, las riquezas provenientes de la minería son repartidas más inclusivamente entre la población trabajadora, además existe menor desempleo en este sector.

Al utilizar como variable dependiente el ratio 20/20, en vez del Gini, se aprecia que el sector silvoagropecuario es significativo y que regiones intensivas en esta actividad tienden a reducir las desigualdades. Se propone por tanto, aumentar la productividad del sector, mejorando la tecnología y eficiencia, permitiendo un mayor ingreso de la población menos favorecida. El papel de la distribución sectorial del PIB, a lo largo del período estudiado, refleja la incidencia del cambio estructural en la distribución de la renta. Esta incidencia dependerá de las fuerzas relativas que puedan estar actuando en direcciones opuestas (tecnologías más intensivas en capital físico o capital humano, enlaces productivos, etc.). Es probable que aquellos sectores con más enlaces productivos apropiados con otras industrias, a nivel regional, contribuyan a mejorar la desigualdad, como es el caso de la minería en Chile.

Otro resultado relevante, es el referido al factor concentración de población indígena. Se concluye que la desigualdad tiende a empeorar en aquellas regiones con mayor concentración de población indígena, respecto al total nacional, lo que enfatiza la desventaja económica de este grupo de la población. El resultado es robusto, ya que se repite en cada una de las estimaciones. Igualmente, la esperanza de vida al nacer refleja que en Chile, las diferencias en acceso a salud de calidad, tienen consecuencias en las desigualdades del ingreso. La variable años de escolaridad promedio, está muy correlacionada con el PIB per cápita, se observa su significancia en la Tabla 3 (utilizando como variable dependiente el ratio 20/20), demostrando que regiones más escolarizadas tienden a reducir las desigualdades. En este sentido, guarda especial importancia la oferta de educación superior, demostrándose en cada caso que las regiones con mayor oferta educativa por habitante, tienden a tener menos desigualdad del ingreso. Se puede inferir, que los esfuerzos de los últimos 13 años para mejorar el acceso a la educación superior, en este caso por medio de la oferta, han contribuido en parte a mejorar los indicadores de desigualdad.

Por consiguiente, se concluye que invertir en capital humano, es decir, mejorar las oportunidades de acceso a la educación y a un sistema de salud de calidad, así como redoblar los esfuerzos para disminuir la brecha entre la población indígena y no indígena, son medidas necesarias para ser un país más inclusivo con sus regiones y reducir las inequidades que tanto mal hacen a un país.

Al considerar el efecto fijo del tiempo, se aprecian diferencias dependiendo del período analizado. El más destacado y cuyo resultado se repite en los distintos modelos, es el período 1996-2000, el cual presenta el mayor coeficiente, siendo positivo y significativo en cada caso. Los resultados reflejaron el alto dinamismo económico disfrutado por el país a partir de la vuelta a la democracia, crecimiento que al parecer, benefició mayormente a la población de más altos ingresos. 
Épocas en que se incrementan más (proporcionalmente) los ingresos de los hogares más ricos, se condicen con períodos de mayor desigualdad. Mientras que, en años donde se incrementan más los ingresos de los hogares más pobres, la desigualdad es menor. Si los esfuerzos políticos se enfocaran en aumentar los ingresos de esta parte de la población (ceteris paribus) se aplacaría más la brecha entre ricos y pobres. Desmintiendo, acorde con distintos autores (Arndt, 1983; Guillén, 2004 y Piketty, 2014) la teoría del "goteo". Cuando no existe ánimo o motivación para reducir las riquezas excesivas de la población de mayores ingresos, a través de política pública que, por ejemplo, obliguen a una mayor participación de los trabajadores en las ganancias de las empresas y una mayor fiscalización de la misma, es muy difícil solucionar el problema de la desigualdad desde este punto. Por tanto, parece ser más factible enfocarse en la parte baja de la distribución, ofreciendo políticas que generen mayores oportunidades, donde las personas no tengan un camino marcado por el solo hecho de nacer en una familia con más o menos riqueza; trabajando en más y mejores políticas educativas y formativas; ejecutando políticas que favorezcan a los sectores menos productivos, y fomentando y facilitando el desarrollo endógeno, sobretodo en las regiones más desiguales.

Al utilizar dos medidas distintas de la distribución del ingreso, podemos comprobar la robustez de los resultados, sobretodo de los factores aporte de sector secundario/minero; concentración de población indígena; esperanza de vida al nacer y educación, como determinantes de la desigualdad del ingreso en Chile. Asimismo, se observa que el ratio 20/20 es más sensible a cambios en las distintas variables analizadas, ya que los modelos tienen un mayor ajuste y los coeficientes de los estimadores, son más altos que los obtenidos utilizando el índice de Gini como variable dependiente.

Dado que las cifras iniciales de desigualdad en Chile son muy altas, los avances son lentos y se necesitaría de un mayor esfuerzo por parte del gobierno y mayor inversión en capital humano, en el sentido amplio. Esta inversión en capital humano, a su vez, implicaría un mayor nivel de PIB futuro, lo que repercutiría indirectamente también en la reducción de la desigualdad.

La principal limitación del estudio, es el bajo número de observaciones, sería ideal poder realizar este análisis a nivel provincial (56 provincias) o comunal (346 comunas), sin embargo, en el país aún no se cuenta con bases de datos robustas a este nivel territorial. Igualmente, en Chile aún es muy difícil encontrar datos a nivel regional de ciertas variables de gran interés en este estudio.

Conociendo las desigualdades que afectan al territorio chileno, el siguiente paso será estudiar el efecto de estas sobre el crecimiento económico regional y por ende nacional. De esta manera, se develará si el exitoso modelo de crecimiento económico actual de Chile será sustentable en el tiempo, o si eventualmente las disparidades afectarán negativamente al progreso económico del país. 


\section{REFERENCIAS}

APARICIO, J. y J. MARQUEZ (2005). "Diagnóstico y especificación de modelos panel en stata 8.0", División de Estudios Políticos-Centro de Investigación y Docencia Económicas, México, pp. 1-11.

ARNDT, H. W. (1983). "The "trickle-down" myth", Economic Development and Cultural Change 32 (1), pp. 1-10.

BANCO CENTRAL DE CHILE (2018). Banco Central de Chile, Información Estadística. 06 de Febrero de 2018, <https://si3.bcentral.cl/Siete/secure/cuadros/home.aspx>.

BANCO MUNDIAL (2018). Banco Mundial, Datos. 10 de Marzo de 2018, <https://datos.bancomundial.org/>.

BARRO, R. (2000). "Inequality and growth in a panel of countries", Journal of economic growth 5 (1), pp. 5-32.

BETANCOUR, M. y P. MALDONADO (Eds.) (2013). Minería en Chile: impacto en regiones y desafíos para su desarrollo, Editorial Salesianos, Santiago de Chile.

BOURGUIGNON, F. y C. MORRISSON (1998). "Inequality and development: the role of dualism", Journal of development economics 57 (2), pp. 233-257.

CONTRERAS, D. (1999). "Distribución del ingreso en Chile. Nueve hechos y algunos mitos", Perspectivas 2 (2), pp. 311-332.

CORREA, V.; A. ESCANDON; R. LUENGO y J. VENEGAS (2002). "Empalme PIB: series anuales y trimestrales 1986-1995, base 1996. Documento metodológico", Banco Central de Chile, Working Paper (179).

DEININGER, K. y L. SQUIRE (1998). "New ways of looking at old issues: inequality and growth", Journal of development economics 57 (2), pp. 259-287.

FORBES, K. (2000). "A reassessment of the relationship between inequality and growth", American economic review 90 (4), pp. 869-887.

GASPARINI, L.; M. CICOWIEZ y W. SOSA (2014). "Pobreza y desigualdad en América Latina: conceptos, herramientas y aplicaciones", Documentos de Trabajo del CEDLAS (171).

GASPARINI, L.; M. MARCHIONNI y W. SOSA (2001). Distribución del ingreso en la Argentina: perspectivas y efectos sobre el bienestar, Triunfar, Córdoba.

GUILLEN, R. (2004). "Revisitando la teoría del desarrollo bajo la globalización", Economía UNAM 1 (1), pp. 19-42.

JIMENEZ, L. y N. RUEDI (1998). "Determinantes de la desigualdad entre los hogares urbanos", Revista de la CEPAL (66), pp. 53-72.

KUZNETS, S. (1955). "Economic growth and income inequality", The American economic review 45 (1), pp. 1-28.

LARRAÑAGA, O. y R. HERRERA (2008). "Los recientes cambios en la desigualdad y la pobreza en Chile", Estudios Públicos 109, pp. 149-186.

MACIAS, A. (2014). "Crecimiento, desigualdad y pobreza: Estado de la cuestión", Revista de economía institucional 16 (31), pp. 101-126.

MARTIN-GUZMAN, M. P. (1988). "Métodos estadísticos en el análisis regional", Revista de estudios regionales (22), pp. 149-170.

MERCHAND, M. A. (2017). "Reflexiones en torno a la nueva geografía económica en la perspectiva de Paul Krugman y la localización de la actividad económica", Breves Contribuciones del IEG -Instituto de Estudios Geográficos "Dr. Guillermo Rohmender"- (21), pp. 206-223.

MIERES, M. (2019). "La dinámica de la desigualdad en Chile: una mirada regional", Working Paper.

MINISTERIO DE DESARROLLO SOCIAL (2015). "Evolución y distribución del ingreso de los hogares (20062013)", Observatorio Social. 12 de Diciembre de 2017, <http://observatorio.ministeriodesarrollosocial. gob.cl/documentos/Casen2013_Evolucion_Distibucion_Ingresos.pdf $>$.

MINISTERIO DE DESARROLLO SOCIAL (2017). "Pueblos indígenas, sístesis de resultados", Observatorio Social. 20 de Febrero de 2018, <http://observatorio.ministeriodesarrollosocial.gob.cl/ casen-multidimensional/casen/docs/CASEN_2015_Resultados_pueblos_indigenas.pdf>.

MINISTERIO DE DESARROLLO SOCIAL (2018). Observatorio Social, Encuesta CASEN. 10 de Noviembre de 2017, <http://observatorio.ministeriodesarrollosocial.gob.cl/index.php>. 
ORGANIZACION PARA LA COOPERACION Y EL DESARROLLO ECONOMICOS (2018). OCDE, Estadísticas. 03 de Marzo de 2018, <https://www.oecd.org/centrodemexico/estadisticas/>.

PEROTTI, R. (1996). Growth, income distribution, and democracy: What the data say. Journal of Economic growth, 1 (2): 149-187.

PIKETTY, T. (2014). El capital en el siglo XXI, Fondo de cultura económica, Santiago de Chile.

SOLIMANO, A. y A. TORCHE (2008). "La distribución del ingreso en Chile, 1987-2006: Análisis y consideraciones de política", Documentos de Trabajo (Banco Central de Chile) (480).

SUNKEL, O. y B. INFANTE (Eds.) (2009). Hacia un desarrollo inclusivo: el caso de Chile, CEPAL, Fundación Chile XXI, OIT, Santiago de Chile.

TODARO, M. y S. SMITH (2012). Economic Development (11 a edición), Addison-Wesley, Boston.

WAGSTAFF, A. (2002). "Pobreza y desigualdades en el sector de la salud", Revista Panamericana de Salud Pública 11 (5), pp. 316-326. 


\section{ANEXOS}

\section{A. Estadística Descriptiva principales variables utilizadas}

\begin{tabular}{|c|c|c|c|c|c|c|}
\hline Variable & Fuente & Obs. & Media & $\begin{array}{l}\text { Desv. } \\
\text { Estándar }\end{array}$ & Mín. & Máx. \\
\hline Gini final & $\begin{array}{l}\text { Ministerio de Desarrollo Social y elaboración de } \\
\text { indicadores en base a Encuesta CASEN }\end{array}$ & 69 & 0,499 & 0,044 & 0,437 & 0,610 \\
\hline Gini inicial & $\begin{array}{l}\text { Ministerio de Desarrollo Social y elaboración de } \\
\text { indicadores en base a Encuesta CASEN }\end{array}$ & 69 & 0,513 & 0,046 & 0,420 & 0,600 \\
\hline LN (PIB p. c.) & $\begin{array}{l}\text { Construido en base a Banco Central de Chile e } \\
\text { Instituto Nacional de Estadísticas }\end{array}$ & 69 & 8,508 & 0,584 & 7,374 & 10,040 \\
\hline LN (Ratio 20/20) & Construido en base a Encuesta CASEN & 69 & 2,317 & 0,257 & 1,896 & 3,043 \\
\hline Aporte sector secundario & Banco Central de Chile & 69 & 24,190 & 9,091 & 4,647 & 47,581 \\
\hline Aporte minería al PIB & Banco Central de Chile & 69 & 15,320 & 18,655 & $-9,32 \mathrm{E}-08$ & 61,533 \\
\hline Concentración población indígena & $\begin{array}{l}\text { Encuesta CASEN e Instituto Nacional de } \\
\text { Estadísticas (1992) (observaciones utilizadas } \\
\text { para el primer período) }\end{array}$ & 69 & 7,308 & 9,616 & 0,348 & 43,374 \\
\hline Esperanza de vida al nacer & $\begin{array}{l}\text { Instituto Nacional de Estadísticas (a través del } \\
\text { Portal de Transparencia del Estado) }\end{array}$ & 69 & 75,040 & 3,770 & 66,360 & 79,630 \\
\hline Años de escolaridad & Encuesta CASEN & 69 & 9,629 & 1,027 & 7,514 & 11,500 \\
\hline
\end{tabular}

Fuente: Elaboración propia.

\section{B. Indices de Gini por regiones 1990-2015}

\begin{tabular}{|l|l|l|l|l|l|l|l|l|l|}
\hline Región & 1990 & 1994 & 1996 & 2000 & 2003 & 2006 & 2009 & 2013 & 2015 \\
\hline Arica y Parinacota & & & & & & & 0,450 & 0,446 & 0,440 \\
Tarapacá & 0,550 & 0,510 & 0,510 & 0,520 & 0,490 & 0,470 & 0,440 & 0,456 & 0,525 \\
Antofagasta & 0,520 & 0,500 & 0,480 & 0,520 & 0,460 & 0,460 & 0,420 & 0,465 & 0,442 \\
Atacama & 0,540 & 0,470 & 0,580 & 0,470 & 0,470 & 0,470 & 0,460 & 0,442 & 0,440 \\
Coquimbo & 0,550 & 0,500 & 0,530 & 0,550 & 0,560 & 0,510 & 0,480 & 0,444 & 0,453 \\
Valparaíso & 0,530 & 0,480 & 0,500 & 0,500 & 0,470 & 0,490 & 0,490 & 0,478 & 0,460 \\
Metropolitana & 0,560 & 0,580 & 0,560 & 0,570 & 0,570 & 0,550 & 0,570 & 0,523 & 0,507 \\
O’Higgins & 0,510 & 0,520 & 0,530 & 0,510 & 0,470 & 0,490 & 0,470 & 0,437 & 0,447 \\
Maule & 0,600 & 0,560 & 0,550 & 0,580 & 0,540 & 0,520 & 0,540 & 0,455 & 0,448 \\
Biobío & 0,580 & 0,530 & 0,570 & 0,600 & 0,550 & 0,530 & 0,510 & 0,465 & 0,474 \\
La Araucanía & 0,590 & 0,590 & 0,550 & 0,610 & 0,590 & 0,550 & 0,580 & 0,497 & 0,502 \\
Los Ríos & & & & & & & 0,520 & 0,494 & 0,506 \\
Los Lagos & 0,580 & 0,550 & 0,550 & 0,530 & 0,550 & 0,520 & 0,520 & 0,500 & 0,470 \\
Aysén & 0,520 & 0,520 & 0,490 & 0,530 & 0,560 & 0,460 & 0,570 & 0,502 & 0,472 \\
Magallanes & 0,530 & 0,450 & 0,490 & 0,570 & 0,510 & 0,500 & 0,510 & 0,448 & 0,456 \\
Promedio & 0,551 & 0,520 & 0,530 & 0,543 & 0,522 & 0,502 & 0,502 & 0,470 & 0,470 \\
\hline
\end{tabular}

Fuente: Indices aportados por el Ministerio de Desarrollo Social y calculados por en autor en base a CASEN. 


\section{Variación (\%) del Ingreso Autónomo del Hogar promedio, primer y último quintil}

\begin{tabular}{|c|c|c|}
\cline { 2 - 3 } \multicolumn{1}{c|}{} & \multicolumn{2}{c|}{ Variación (\%) ingreso autónomo del hogar } \\
\hline Período & Primer quintil & Ultimo quintil \\
\hline $1990-1994$ & 97,7 & 98,6 \\
$1996-2000$ & 22,2 & 28,7 \\
$2003-2006$ & 21,7 & 9,9 \\
$2009-2013$ & 60,6 & 10,6 \\
$2013-2015$ & 18,9 & 10,6 \\
\hline
\end{tabular}

Fuente: Elaboración propia basada en datos CASEN.

\section{Resumen resultados socioeconómico regionales}

\begin{tabular}{|c|c|c|c|c|c|c|}
\hline \multirow{2}{*}{$\begin{array}{c}\text { Variables } \\
\text { (considera } 15 \text { regiones) }\end{array}$} & \multicolumn{3}{|c|}{ Mejores } & \multicolumn{3}{|c|}{ Peores } \\
\hline & $1^{\circ}$ & $2^{\circ}$ & $3^{\circ}$ & $1^{\circ}$ & $2^{\circ}$ & $3^{\circ}$ \\
\hline PIB per cápita 2015 & Antofagasta & Atacama & Tarapacá & La Araucanía & $\begin{array}{c}\text { Arica y } \\
\text { Parinacota }\end{array}$ & Maule \\
\hline IDH 2003 & $\mathrm{RM}$ & Magallanes & Tarapacá & Maule & La Araucanía & Los Lagos \\
\hline $\begin{array}{l}\text { IDERE } \\
\text { (6 dimensiones) } 2015\end{array}$ & RM & Magallanes & Antofagasta & La Araucanía & Maule & Aysén \\
\hline $\begin{array}{l}\text { IDERE } \\
\text { (7 dimensiones) } 2015\end{array}$ & $\mathrm{RM}$ & Magallanes & Valparaíso & La Araucanía & Maule & Aysén \\
\hline Pobreza 2015 & Magallanes & Antofagasta & Aysén & La Araucanía & Maule & Biobío \\
\hline $\begin{array}{l}\text { Pobreza } \\
\text { Multidimensional } 2015\end{array}$ & Magallanes & Aysén & Antofagasta & La Araucanía & Atacama & Coquimbo \\
\hline Indice 10/10 2015 & $\begin{array}{l}\text { Arica y } \\
\text { Parinacota }\end{array}$ & Antofagasta & Atacama & La Araucanía & Los Ríos & Los Lagos \\
\hline Indice 20/20 2015 & $\begin{array}{l}\text { Arica y } \\
\text { Parinacota }\end{array}$ & Atacama & Antofagasta & La Araucanía & Los Ríos & Biobío \\
\hline $\begin{array}{l}\text { Ingreso Autónomo del } \\
\text { Hogar } 2015\end{array}$ & $\mathrm{RM}$ & Antofagasta & Tarapacá & La Araucanía & Maule & Los Lagos \\
\hline Gini (met. nueva) 2015 & $\begin{array}{c}\text { Arica y } \\
\text { Parinacota }\end{array}$ & Atacama & Antofagasta & La Araucanía & $\mathrm{RM}$ & Los Ríos \\
\hline $\begin{array}{l}\text { Años de Escolaridad } \\
\text { Promedio } 2015\end{array}$ & $\mathrm{RM}$ & $\begin{array}{l}\text { Tarapacá y } \\
\text { Antofagasta }\end{array}$ & $\begin{array}{c}\text { Arica y } \\
\text { Parinacota }\end{array}$ & $\begin{array}{c}\text { Maule y } \\
\text { Los Lagos }\end{array}$ & La Araucanía & $\begin{array}{l}\text { O’Higgins y } \\
\text { Los Ríos }\end{array}$ \\
\hline \% Analfabetismo 2015 & Tarapacá & Magallanes & Antofagasta & Maule & La Araucanía & Los Ríos \\
\hline Esperanza de Vida 2015 & Coquimbo & $\mathrm{RM}$ & Atacama & Los Lagos & Antofagasta & Magallanes \\
\hline $\begin{array}{l}\text { Especialistas } / 10.000 \\
\text { hab. } 2015\end{array}$ & $\mathrm{RM}$ & Magallanes & Aysén & Maule & Atacama & Tarapacá \\
\hline
\end{tabular}

Fuente: Elaboración propia. 


\section{E. DEFINICIONES}

Años Escolaridad Promedio: Años de escolaridad personas con 15 años o más. Obtenido de la Encuesta CASEN.

Aporte Sector Primario al PIB 2009: Aporte al PIB regional, de los sectores silvoagropecuario, pesca y minería. Calculado con base en datos Banco Central de Chile.

Aporte Sector Secundario al PIB 2009: Aporte al PIB regional, de los sectores industria manufacturera; electricidad, agua y gas y sector construcción. Calculado en base a datos Banco Central de Chile.

Aporte Sector Terciario al PIB 2009: Aporte al PIB regional, de los sectores comercio, restaurantes y hoteles; transporte, información y comunicaciones; servicios financieros y empresariales; servicios de vivienda e inmobiliarios; servicios personales y administración pública, 2009. Calculado en base a datos Banco Central de Chile.

Concentración de Población Indígena: Porcentaje de población que declara pertenecer a alguna etnia, presente en una región, respecto al total nacional. Calculado con base en la Encuesta CASEN.

Esperanza de Vida al nacer: Años promedio de esperanza de vida al nacer. Obtenido de INE Chile.

Indice de Gini: "El índice de Gini mide hasta qué punto la distribución del ingreso (o, en algunos casos, el gasto de consumo) entre individuos u hogares dentro de una economía se aleja de una distribución perfectamente equitativa". "Un índice de Gini de 0 representa una equidad perfecta, mientras que un índice de 100 representa una inequidad perfecta" (Banco Mundial, 2018). Obtenido de Resultados Encuesta CASEN (años 2013 y 2015 calculado por el autor).

Ingreso Autónomo del Hogar: "Es la suma de todos los pagos que reciben todos los miembros del hogar, excluido el servicio doméstico puertas adentro, provenientes tanto del trabajo como de la propiedad de los activos. Estos incluyen sueldos y salarios, monetarios y en especies, ganancias provenientes del trabajo independiente, la autoprovisión de bienes producidos por el hogar, rentas, intereses, dividendos y retiro de utilidades, jubilaciones, pensiones o montepíos, y transferencias corrientes" (Ministerio de Desarrollo Social, 2015, pp. 6). Obtenido de la Encuesta CASEN.

Ingreso Primer Quintil: Corresponde al ingreso autónomo del hogar del 20\% de la población de menores ingresos. Calculado en base a la Encuesta CASEN. 
Ingreso Último Quintil: Corresponde al ingreso autónomo del hogar del 20\% de la población de mayores ingresos. Calculado con base en la Encuesta CASEN.

Oferta de Educación Superior: Número de vacantes en instituciones de educación superior por cada mil habitantes. Las instituciones pueden ser: Universidades públicas o privadas, centros de formación profesional, institutos profesionales o centros educativos de las Fuerzas Armadas de Chile. Información facilitada por el Consejo Nacional de Educación.

PIB per cápita: Es el Producto Interno Bruto por cada habitante. Obtenido con base en el (Banco Central de Chile, 2018). Se calculó utilizando el método de la tasa de variación, de forma tal de unir una serie de datos del PIB por región, a precios constantes, base 1986, 1996, 2003 y PIB, volumen a precios del año anterior encadenado, series empalmadas, referencia 2013, llevando los valores a este último año.

Ratio 20/20: "Indice de desigualdad que muestra la relación entre el ingreso recibido por el 20\% de hogares de mayores ingresos y el correspondiente al 20\% de hogares con menores ingresos" (Ministerio de Desarrollo Social, 2015, pp. 37). Calculado con base en la Encuesta CASEN. 
